\title{
EFECTIVIDAD A NIVEL IN VITRO DE Trichoderma spp. NATIVOS E IMPORTADOS CONTRA Fusarium oxysporum*
}

\author{
Daniela Rodríguez-García ${ }^{1 / * *}$, Amy Wang-Wong ${ }^{2}$ \\ Palabras clave: Porcentaje de inhibición del crecimiento radial; antagonista; cepas nativas; \\ control biológico; micoparasitismo. \\ Keywords: Percentage of radial growth inhibition (PRGI); antagonist; native strains; \\ biological control; mycoparasitism.
}

Recibido: 08/07/2019

RESUMEN

Introducción. Los hongos fitopatógenos causan pérdidas en la producción de tomate y por ello es importante y necesaria la búsqueda de alternativas orientadas al manejo de agentes antagonistas que sean eficientes y compatibles con el ambiente. Objetivo. Determinar la efectividad de cepas nativas e importadas de Trichoderma spp. en el combate de Fusarium spp. a nivel de laboratorio. Materiales y métodos. Se emplearon productos comerciales de Trichoderma spp. con cepas nativas (THM-03 y THM-04) e importadas (THU-01 de Estados Unidos y THC-02 de Colombia). El patógeno fue aislado de plantas enfermas de tomate, para los cuales se empleó análisis molecular y morfológico para su identificación. Se utilizó la técnica de cultivo dual para evaluar los radios de crecimiento del antagonista (RCA) y del patógeno (RCP), el micoparasitismo y el porcentaje de inhibición del crecimiento radial (PICR). Resultados. Se identificaron las especies $T$. asperellum, $T$.

\footnotetext{
* Esta investigación formó parte de la tesis para grado de maestría académica de la primera autora.

** Autora para correspondencia. Correo electrónico: daniela.rodriguez@ucr.ac.cr
}

Aceptado: 19/11/2019

\section{ABSTRACT}

In vitro effectiveness of Trichoderma spp. native and imported against Fusarium oxysporum. Introduction. Phytopathogenic fungi cause losses in tomato production and for this reason it is important and necessary to find alternatives aimed at managing antagonistic agents that are efficient and compatible with the environment. Objective. To determine the effectiveness of native and exotic strains of Trichoderma spp. against Fusarium spp. at laboratory level. Materials and methods. Commercial products of Trichoderma spp. with native (THM-03 and THM-04) and imported strains (THU-01 from the United States and THC02 from Colombia) were used. Pathogen was isolated from diseased tomato plants, for which morphological and molecular analyses were used for identification. Dual culture technique was used to evaluate radial growth of the antagonist (RGA) and the pathogen, mycoparasitism and percentage of radial growth inhibition (PRGI).

1 Universidad de Costa Rica, Centro de Investigaciones Agronómicas, Laboratorio de Microbiología Agrícola, Costa Rica. (iD) 0000-0003-0541-2488.

2 Universidad de Costa Rica, Centro de Investigación en Protección de Cultivos, Instituto de Investigaciones Agrícolas, Costa Rica. (iD) 0000-0002-6149-3035. 
asperelloides y T. guizhouense; el patógeno se identificó como $F$. oxysporum. Los valores promedios de los RCA fueron 26,$5 ; 39,3 ; 41,2$ y $32,1 \mathrm{~mm}$ para THU-01, THC-02, THM-03 y THM-04, respectivamente, y $42,75 \mathrm{~mm}$ para los testigos. En cuanto al micoparasitismo, se obtuvo que THC-02, THM-03 y THM-04 presentaron invasión completa de la colonia del patógeno con esporulación y THU-01 solo logró invadir un cuarto de la superficie del patógeno. Se determinó el PICR, en el que se encontraron diferencias entre cepas donde las más efectivas fueron THM-3 y THC-2 con $67 \%$ y $63 \%$ de inhibición, respectivamente. Conclusión. En este estudio in vitro se encontraron diferencias en la capacidad antagónica de las especies de Trichoderma frente al patógeno $F$. oxysporum. Las cepas $T$. asperellum, T. asperelloides y la cepa nativa de T. guizhouense mostraron ser más agresivas en el combate del patógeno, independientemente de su procedencia, por lo que se podría ampliar el estudio con el uso de más aislamientos.

\section{INTRODUCCIÓN}

En la actualidad, no se conoce de algún método eficiente que por sí solo disminuya significativamente la enfermedad de la marchitez causada por Fusarium spp. en diferentes cultivos. Se han realizado grandes esfuerzos por combatirla $\mathrm{y}$ en estos momentos varios países aplican un manejo integrado, en el que se incluyen la poda fitosanitaria, fertilización al suelo, reguladores de crecimiento y aspersiones con fungicidas (Jiménez y Sanabria 1997a y b, Agrios 2005, Caravaca 2008). Estos últimos son utilizados con mayor frecuencia; sin embargo, su uso indiscriminado provoca problemas de contaminación ambiental y en general, desequilibrio ecológico (De la Cruz 2004).
Results. T. asperellum, T. asperelloides and T. guizhouense were identified; the pathogen was identified as $F$. oxysporum. Mean RGA values of $25.6,39.3,41.2$ and $32.1 \mathrm{~mm}$ were obtained for THU-01, THC-02, THM-03 and THM-04, respectively; and $42.75 \mathrm{~mm}$ for the control. Regarding mycoparasitism, THC-02, THM-03 and THM-04 presented complete invasion of the $F$. oxysporum colony, with sporulation and THU-01 only managed to invade a quarter of the pathogen's surface. PRGI was determined, in which significant differences were found between Trichoderma strains where the most effective strains were THM-03 and THC-02 with 67 and 63\% inhibition, respectively. Conclusion. This in vitro study showed differences in the antagonistic capacity of different Trichoderma species against the pathogen $F$. oxysporum. Strains of $T$. asperellum (THC-02), $T$. asperelloides (THM-03) and the native strain T. guizhouense (THM-04) showed to be more aggressive in combat of the pathogen, regardless of its origin, thus the study could be expanded with the use of more isolates.

El uso de Trichoderma como agente de biocontrol representa una alternativa viable, por ser eficaz, fácil de producir a gran escala, la mayoría son especies cosmopolitas y presentan gran capacidad de parasitar; además compiten por nutrientes o producen compuestos que resultan antagónicos para una gran variedad de hongos fitopatógenos tales como los géneros Rhizoctonia spp., Fusarium spp., Pythium spp., entre otros (Montealegre et al. 2005, Chérif y Benhamou 1990, Córdoba 2003, Hibar et al. 2005, 2006, Gupta et al. 2014).

Los eventos que conllevan al micoparasitismo son complejos y tienen lugar de manera ordenada. En primer lugar, Trichoderma localiza al patógeno y comienza a crecer por tropismo hacia él (Lu et al. 2004). Esta detección a 
distancia se debe al menos parcialmente, a la expresión secuencial de enzimas que degradan la pared celular de los hongos, en inglés conocido como Cel Wall Degrading Enzymes (CWDEs). Aunque las diferentes cepas de Trichoderma pueden seguir distintos patrones de inducción de CWDEs, parece seguro que una exoquitinasa extracelular, que se produce de forma constitutiva en pequeñas cantidades, podría estar implicada en este proceso. Esta exoquitinasa provoca la liberación de algunos oligómeros de la pared del hongo. Esos compuestos son los que inducen la expresión de endoquitinasas tóxicas (Brunner et al. 2003, Harman 2006) que al ser liberadas por Trichoderma, se difunden y comienzan el ataque al hongo patógeno antes de que se haya producido el contacto físico (Zeilinger et al. 1999, Viterbo et al. 2002b).

Es importante mencionar que el mecanismo micoparasítico de Trichoderma integra la acción de diversas CWDEs entre las cuales se encuentran quitinasas, $\beta$-1,3-glucanasas, $\beta-1,6$ glucanasas y proteasas (Elad et al. 1982, Papavizas 1985, Chet et al. 2002, Sanz et al. 2004).

Debido a que la quitina y el $\beta$-1,3-glucano son los principales componentes estructurales de la pared celular de hongos, se ha propuesto que las enzimas claves en este proceso son las quitinasas y las $\beta$-1,3-glucanasas (Elad et al. 1982, Papavizas 1985, De la Cruz et al. 1993). Por otra parte, se tienen en cuenta otras enzimas que hidrolizan otros componentes minoritarios de las paredes fúngicas (proteínas, $\beta$-1,6-glucanos, $\alpha$-1,3-glucanos) (Viterbo et al. 2002a, Sanz et al. 2005, Suárez et al. 2008, Olmedo y Casa 2014).

Actualmente, en Costa Rica, no se dispone de información certera respecto a las diferentes cepas de Trichoderma spp. aisladas de los mismos sitios de cultivo, o si las importadas de otros cultivos o países, presentan la misma eficiencia y especificidad en el combate de las especies del género Fusarium. Además, aún se tienen interrogantes acerca de la existencia de alguna relación específica antagónica entre Trichoderma spp. y Fusarium spp., o si todas las cepas tienen potencial de antibiosis, por lo que es requerido detectar su presencia para conocer la diversidad de cepas nativas, para evaluarlas como agentes potenciales de combate biológico y comprobar su especificidad con respecto al patógeno a combatir (Papavizas 1985, Nelson 1991). Por lo anterior, se propuso como objetivo de este análisis, determinar la efectividad de cepas nativas e importadas de Trichoderma spp. en el combate de Fusarium spp. a nivel de laboratorio.

\section{MATERIALES Y MÉTODOS}

Ubicación. El estudio se realizó en el Laboratorio de Microbiología Agrícola, del Centro de Investigaciones Agronómicas de la Universidad de Costa Rica.

Aislamiento e identificación de las cepas de Trichoderma spp. y del patógeno Fusarium spp. Se emplearon productos comerciales preparados con diferentes cepas de Trichoderma spp. provenientes de Estados Unidos (THU-01) y Colombia (THC-02); así como 2 cepas nativas de Costa Rica pertenecientes a la Colección de Microorganismos Benéficos del Área de Biocontrol, del Laboratorio de Microbiología Agrícola del Centro de Investigaciones Agronómicas de la Universidad de Costa Rica (THM-03, San Carlos y THM-04, Cartago).

Los aislamientos de Trichoderma spp. a partir de los productos comerciales se realizaron mediante el método de diluciones seriadas en caja Petri, para lo cual se tomó una muestra de 1 gr del producto y se diluyó en $10 \mathrm{~mL}$ (proporción $1 / 10 \mathrm{p} / \mathrm{v}$ ) de agua destilada estéril hasta obtener diluciones de $10^{-2}$ y $10^{-3}$. De la última dilución, se extrajo una alícuota de $0,1 \mathrm{~mL}$ y se distribuyó homogéneamente sobre una caja Petri, que contenía medio de cultivo agar papa dextrosa (PDA). Se incubaron a $25 \pm 1^{\circ} \mathrm{C}$ por 5-6 días en oscuridad y luego 4 días con luz día en una incubadora Digisystem DK-500. Se utilizaron 10 cajas Petri por cada producto comercial. Para la identificación se utilizó el método descrito por Gilchrist et al. (2005), y las claves de identificación propuestas por Samuels et al. (2013) y Ellis (2006). 
Los aislamientos de Fusarium spp., se realizaron a partir de muestras de plantas enfermas de tomate que presentaban marchitez, necrosamiento basal del tallo y de las axilas de las ramas laterales inferiores. Las plantas se lavaron con agua común para eliminar el exceso de suelo presente en el tallo y raíz. De los tejidos se hicieron disecciones de $0,5 \mathrm{~cm}$ de la zona de avance de la enfermedad, cada trozo se desinfectó con hipoclorito de sodio al 2,5\% por $2 \mathrm{~min}$ y, posteriormente, se realizó 4 enjuagues con agua destilada estéril. Luego, cada trozo se fraccionó en secciones de aproximadamente $0,25 \mathrm{~cm}$ y se colocaron en cajas Petri con medio de cultivo papa dextrosa agra (PDA). Los aislamientos obtenidos se purificaron mediante la técnica de punta de hifa descrita por Chairman et al. (1978), la cual consiste en seleccionar la punta de una hifa solitaria, cortarla y transferirla a una caja Petri. Se usó medio de cultivo Komoda (Komoda 1975) y se dejó incubar por 2 o 3 días.

Las diferentes cepas obtenidas se identificaron morfológicamente con base en el protocolo del Fusarium Research Center de la Universidad de Pennsylvania, desarrollado por Burges et al. (1994) y Gilchrist et al. (2005), y las claves taxonómicas de Booth (1971), Arikan et al. (2001) y Seifert (1996).

\section{Caracterización molecular de las cepas} de Trichoderma spp. y Fusarium spp. El ensayo se llevó a cabo en el Laboratorio de Técnicas Moleculares del CIPROC de la Facultad de Ciencias Agroalimentarias de la Universidad de Costa Rica. Se realizó la extracción del ADN de las diferentes cepas de Trichoderma spp. y de los aislamientos de $F$. Oxysporum; para esto, se separó un disco $5 \mathrm{~cm}$ de diámetro de un cultivo de 3-5 días de crecimiento de los hongos. Luego los discos de micelio se secaron con toallas de papel absorbente. Posteriormente, el disco se colocó en un tubo Eppendorf de 1,5 mL para la extracción de ADN. La extracción de ADN genómico se realizó utilizando el kit de aislamiento de ADN Genómico de Puregene ${ }^{\mathrm{TM}}$.

Posteriormente, para Trichoderma spp., se llevó a cabo la amplificación por PCR de un fragmento de $0,9 \mathrm{~kb}$ del extremo $5^{\prime}$ del factor de elongación $-1 \alpha$ (TEF) del gen (eEFla1) que contiene 3 intrones que fueron amplificados utilizando el par de imprimador tef71f (CAAAATGGGTAAGGAGGA(G/C)AAGAC) y el tef997R (CAGTACCGGC(A/G) GC(A/G) AT(A/G)AT(G/C)AG) (Shoukouhi y Bissett 2008). Luego se hizo una electroforesis para corroborar el resultado de la amplificación y, por último, el producto de PCR obtenido se limpió empleando el kit de purificación para PCR (QIAGEN), se envió a secuenciar a la empresa Macrogen Inc., en Seúl, Corea.

Para el caso de los aislamientos de Fusarium spp., se efectuó el protocolo para el PCR para amplificar la región del gen TEF, utilizando el imprimador de inicio ef1 (5'-TGGGTAAGGA(A/G)GACAAGAC-3) y el imprimador de reversa ef2 (5'GGA(G/A) GTACCAGT(G/C)ATCATGTT-3') (O’Donnell et al. 1998, Geiser et al. 2004); a los productos amplificados del hongo se les efectuó una electroforesis en gel de agarosa. Finalmente, se realizó la secuenciación por la empresa Macrogen Inc., en Seúl, Corea.

Las secuencias obtenidas para cada imprimador fueron analizadas con el programa Bioedit Sequence Alignment Editor version 7.0.5.3 (Hall 1999) y se obtuvo la secuencia consenso. Las secuencias se compararon con las secuencias publicadas en la base de datos del Banco de Genes del Centro Nacional para la Información Biotecnológica en USA (NCBI), mediante la opción BLASTn search (Altschul et al. 1997).

Se alinearon con el programa Clustal W (Thompson et al. 1994) la secuencia de ADN de la región del factor de elongación - $1 \alpha$ (TEF) del gen (eEF1a1) de las cepas de Trichoderma spp. e incluidas las secuencias de aislamientos de especies de Trichoderma del Banco de Genes. 
El alineamiento fue corregido manualmente en el programa BioEdit. El análisis filogenético de las secuencias se hizo con el programa MEGA 6 (Tamura et al. 2013), según el criterio de Máxima Verosimilitud, el modelo Kimura 2-parameter y un análisis de 2000 réplicas de Bootstrap. Se incluyó la secuencia de Trichoderma aggressivum CBS 100.525 (AF534614) como grupo externo (raíz) en el análisis filogenético de las especies del complejo de Trichoderma harzianum. Las secuencias de la región del factor de elongación -1 $\alpha$ (TEF) utilizadas fueron las mismas que empleó Chaverri et al. (2015) en su análisis filogenético del Complejo de T. harzianum.

Pruebas de micoparasitismo a nivel de laboratorio. Se utilizó la técnica del papel celofán modificada (Dennis y Webster 1971), para seleccionar aquellas cepas de Trichoderma spp. que presentaron capacidad antagónica in vitro, al inhibir o retardar el crecimiento del micelio de Fusarium spp. Se cortaron tiras de 2,0 cm de ancho por $8,5 \mathrm{~cm}$ de largo de papel celofán, las cuales se esterilizaron antes de colocarlas bajo condiciones asépticas dentro de la caja Petri, sobre el medio de cultivo PDA. Posteriormente, en un extremo de la caja de Petri, se colocó un disco de agar de $4 \mathrm{~mm}$ de diámetro con micelio de Fusarium spp. de 8 días de edad y se dejó desarrollar durante 3 días por su crecimiento lento. Luego, en el extremo opuesto otro disco de $4 \mathrm{~mm}$ con micelio de Trichoderma spp. (nativos o comerciales) de la misma edad, a una distancia aproximada de $5 \mathrm{~cm}$ entre ellos (Howell 2003). También se cultivó, por separado cada antagonista y patógeno; se colocó un disco de agar de $4 \mathrm{~mm}$ de diámetro de micelio en el centro de la caja Petri, que correspondieron a los testigos y se incubaron bajo las condiciones antes descritas.

Se midió cada $24 \mathrm{~h}$ el crecimiento radial del micelio de la colonia de los hongos, hasta observar el traslape de hifas de ambos hongos en el papel celofán. En este momento se retiró el papel y se observó con microscopio (Biológico Binocular, Modelo Xsz 206 B) con aumento de $40 \mathrm{x}$, para determinar si se presentaba hiperparasitismo.

Para evaluar la capacidad antagónica o antagonismo de aislamientos nativos o foráneos de Trichoderma spp., se utilizó la técnica de Cherif y Benhamou (1990), y cada tratamiento se depositó en un extremo de cajas Petri con PDA, un disco de $5 \mathrm{~mm}$ de diámetro con micelio activo de colonias fungosas de 8 días de edad de Fusarium spp. y se dejaron desarrollar durante 3 días por su crecimiento lento. Posteriormente, en el otro extremo de la caja se depositaron discos de $5 \mathrm{~mm}$ de Trichoderma spp. Luego se incubaron a $25 \pm 1^{\circ} \mathrm{C}$ por 5-6 días en oscuridad y luego, 4 días con luz de día en una incubadora Digisystem DK-500. Se midió cada $24 \mathrm{~h}$ el crecimiento radial del micelio de la colonia de los hongos.

Se comprobó de distintas formas: a) el grado de micoparasitismo, mediante la escala propuesta por Ezziyyani et al. (2004) (Tabla 1).

Tabla 1. Escala para evaluar del grado de micoparasitismo según Ezziyyani et al. (2004).

\begin{tabular}{cl}
\hline Grado & Capacidad antagónica \\
\hline 0 & Ninguna invasión de la superficie de la colonia del hongo patógeno \\
1 & Invasión de $1 / 4$ de la superficie de la colonia del hongo patógeno \\
2 & Invasión de $1 / 2$ de la superficie de la colonia del hongo patógeno \\
3 & Invasión total de la superficie de la colonia del hongo patógeno, no esporulación sobre ella \\
4 & Invasión total de la superficie de la colonia del hongo patógeno esporulación sobre ella \\
\hline
\end{tabular}


Además, se evaluó: b) la competencia por nutrientes y espacio, la cual se obtuvo con los radios de crecimiento de cada patógeno y antagonista en cultivo dual, junto con sus respectivos testigos, utilizando un calibrador "Pie de rey", y c) por el porcentaje de inhibición de crecimiento radial (PICR) con la fórmula utilizada por Ezziyyani et al. (2004).

$$
\mathrm{PICR}=(\mathrm{R} 1-\mathrm{R} 2) / \mathrm{R} 1 \times 100
$$

$\mathrm{R} 1$ = radio mayor (radio patógeno testigo).

$\mathrm{R} 2$ = radio menor (radio del patógeno en enfrentamiento con el antagonista).

El análisis estadístico que se utilizó fue un análisis de varianza (Anova) y prueba de comparación de medias de Tukey $(\mathrm{p}<0,05)$ con el programa estadístico Infostat versión 6.12, bajo un diseño experimental completamente al azar con 3 repeticiones que constaban de un plato Petri, como unidad experimental, y 4 réplicas biológicas por cepa de Trichoderma spp. Se midieron las siguientes variables: radio de crecimiento del antagonista (RCA), radio de crecimiento del patógeno (RCP), micoparasitismo (MICMO) y PICR.

\section{RESULTADOS}

Aislamiento e identificación de las cepas de Trichoderma spp. y del patógeno Fusarium spp. Las cepas THU-01 (Estados Unidos) y la cepa nativa THM-04 se identificaron dentro del complejo de Hypocrea lixii/ Trichoderma harzianum (Figura 1), sin embargo, nuevos estudios filogenéticos identifican estas cepas como Trichoderma guizhouense (Chaverri et al. 2015) (Figura 2). Las mismas crecieron muy rápido en medio de cultivo PDA y mostraron un aspecto polvoso. Al inicio, las colonias presentaron una coloración blanco amarillento que luego se tornó verde. Con microscopio, se observaron fialides terminales en un verticilo o solitarias de forma ampuliformes, por lo general, fuertemente constreñidos por debajo de la punta y más largas que las fialides subterminales con un conidio sucesivo en la extremidad y conidias de forma globosa a subglobosa de color verde olivo, características que concuerdan con las descritas por Chaverri et al. (2015) para T. guizhouense. 


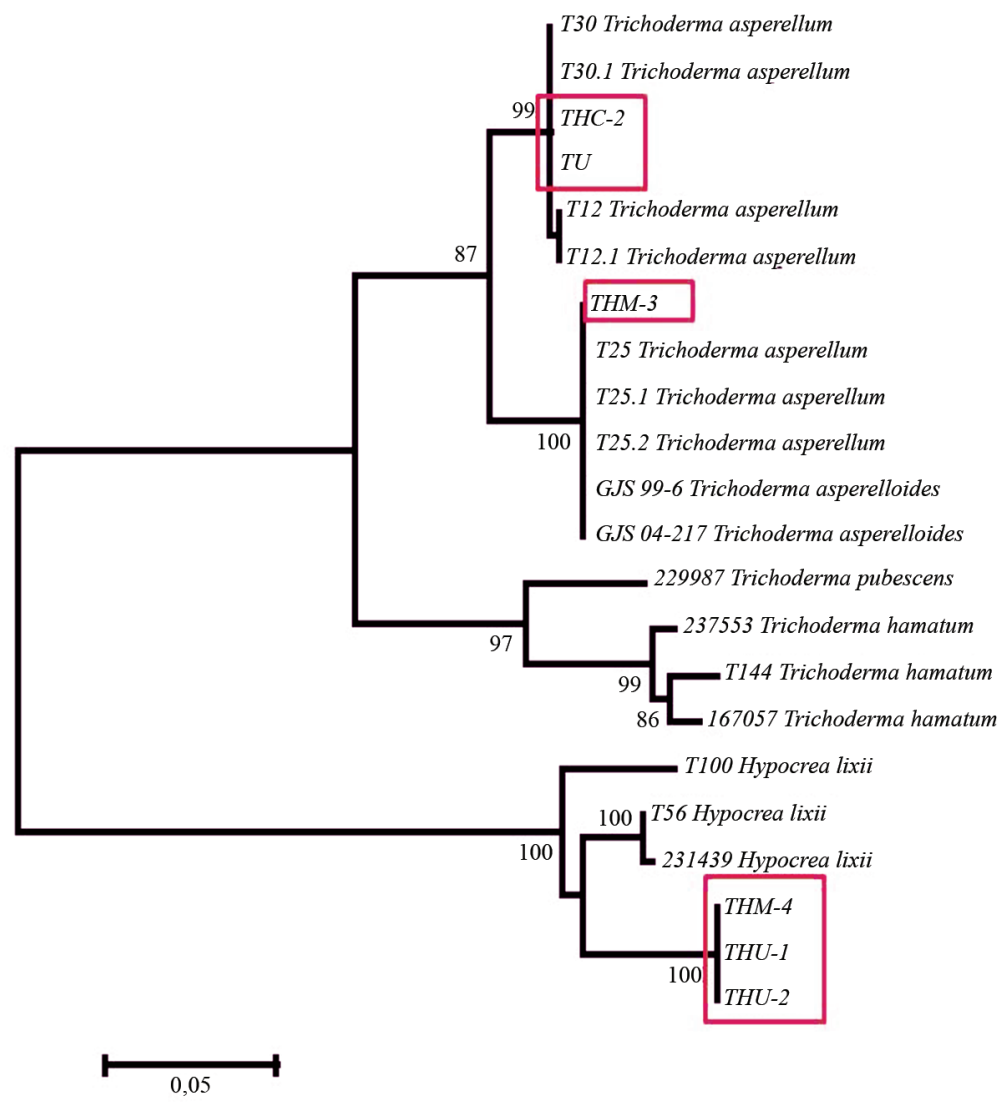

Figura 1. Relación filogenética de especies de Trichoderma nativas e importadas en productos comerciales con base en la secuencia del factor de elongación $-1 \alpha(t e f)$ gen (eEF1a1).

Árbol construido con el programa MEGA 6 software, método Máxima Verosimilitud (ML), modelo Kimura 2-parameter y 2000 réplicas de Bootstrap. Valores de Bootstrap se indican en los nodos. 


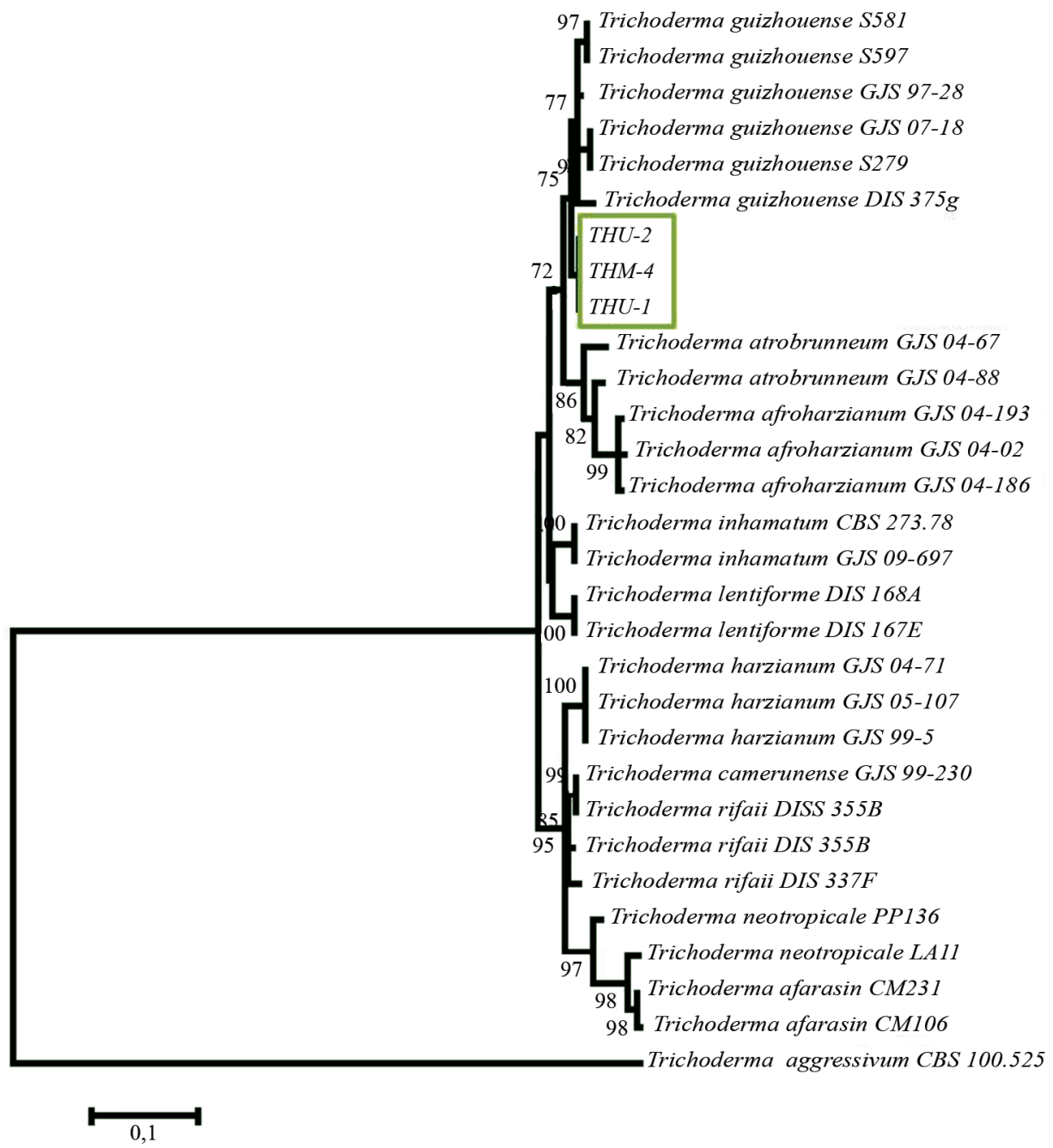

Figura 2. Relación filogenética de especies de Trichoderma harzianum nativas e importadas en productos comerciales con base en la secuencia del factor de elongación -1 $\alpha$ (tef) gen (eEF1a1).

Árbol construido con el programa MEGA 6 software, método Máxima Verosimilitud (ML), modelo Kimura 2-parameter y 2000 réplicas de Bootstrap.

Se incluyó de raíz a $T$. aggressivum.

Valores de Bootstrap se indican en los nodos.

Al colocar las cepas THC-02 (Colombia) y la cepa nativa THM-03 en medio de cultivo PDA, crecieron formaron anillos concéntricos, los primeros en crearse fueron de color verde oscuro, mientras que en los márgenes el micelio fue blanco y no se observó pigmentación amarilla.
Con microscopio se observaron fialides con aspecto de candelabro producidos en las puntas de las hifas, ligeramente más grandes en el medio y levemente constreñida en la base, con conidias subglobosas a elipsoidales de color verde oscuro, con clamidosporas normalmente abundantes 
y subglobosas. De acuerdo con lo descrito por Samuels et al. (2010, 2013), las cepas THC-02 y THM-03 pertenecen a Trichoderma asperellum.

A partir de plantas enfermas de tomate, se aislaron 2 cepas de Fusarium sp., las cuales se caracterizaron por las colonias que crecieron en el medio de cultivo, y que expusieron un micelio aéreo blanco, que posteriormente adquirió, en uno de los aislamientos, una coloración púrpura (F1), mientras que el otro aislamiento presentó esporodoquios de coloración anaranjado medio (F2). Ambas cepas presentaron conidióforos monofialides cortos, simples, laterales en el micelio aéreo, más tarde en forma de racimos densamente ramificados. Los macroconidios eran fusiformes, principalmente, de 3 septos y abundantes microconidios elipsoidales, no septados en su mayoría. Las clamidosporas eran terminales o intercalares e hialinas. Las características identificadas concuerdan con las descritas por Burges et al. (1994) y Gilchrist et al. (2005) y las claves taxonómicas de Booth (1971), Arikan et al. (2001), y Seifert (1996) con F. oxysporum.

En lo siguiente, fue seleccionada la cepa F1 de $F$. oxysporum para llevar a cabo los demás ensayos, debido a que la cepa F2 presentó problemas de crecimiento en los diferentes medios de cultivo utilizados.

Caracterización molecular de las cepas de Trichoderma spp. y Fusarium spp. Las secuencias de la región del factor de elongación $-1 \alpha$ (TEF) obtenidas de las diferentes cepas aisladas de Trichoderma, de los productos nativos e importados, se alinearon con secuencias encontradas en el Banco de Genes (Gen Bank) (Tabla 2); se identificaron THU-1 y THM-4 con un $97 \%$ de similitud con T. harzianum/ H. lixii. mientras que en el caso de THC-2 tuvo un $99 \%$ de similitud con T. asperellum y el mismo porcentaje obtuvo THM-3 con T. asperelloides.

Tabla 2. Identificación de las cepas de Trichoderma spp. nativas e importadas presentes en los productos evaluados con base en la secuencia parcial del gen TEF según Gen Bank.

\begin{tabular}{lclc}
\hline Producto & Similitud (\%) & \multicolumn{1}{c}{ Identidad } & Accesión Gen Bank \\
\hline THU-01 & 97 & Complejo T. harzianum & EF191330 \\
THC-2 & 99 & T. asperellum & KP262481 \\
THM-03 & 99 & T. asperelloides & KP696458 \\
THM-04 & 97 & Complejo T. harzianum & EF191330 \\
\hline
\end{tabular}

Al realizar el análisis filogenético basado en los fragmentos de tef (Figura 1), se demostró que las cepas THU-01 y THM-04, con un valor de Boostrap de 100, eran idénticas, por lo que formaron un grupo monofilético dentro del complejo de T. harzianum/H. lixii.

Por otra parte, en el árbol de la Figura 1 se muestra también que existió una relación entre las especies de T. asperellum y T. asperelloides con un Boostrap de 87, sin embargo, con un Boostrap de 99 se encontró la cepa THC-02 dentro del grupo de T. Asperellum, y con un Boostrap del 100 se identificó el grupo de $T$.
Asperelloides, así como algunos aislamientos de T. asperellum que no se han reclasificado.

Complementariamente, se realizó otro análisis filogenético para determinar la correcta clasificación de las cepas de Trichoderma que en el primer análisis fueron agrupados dentro del complejo de T. harzianum/ H. lixii, y se obtuvo que corresponden a la nueva especie identificada como Trichoderma guizhouense (Figura 2).

Al comparar las cepas aisladas de Fusarium spp., las secuencias obtenidas de la región del factor de elongación $-1 \alpha$ (TEF) con otras en el Banco de Genes, se encontró una similitud 
del $99 \%$ para la cepa F1; mientras que, la cepa F2 solo obtuvo una similitud del 98\%, las cuales se identificaron como $F$. oxysporum con número de Accesión KF574861 y KJ648634, respectivamente.

Pruebas de micoparasitismo a nivel de laboratorio. Al realizarse la confrontación in vitro de las diferentes cepas aisladas de productos comerciales importados (THU-01 y THC-02) y nativas (THM-03 y THM-04) de Trichoderma spp. frente al patógeno Fusarium oxysporum, estas presentaron radios de crecimiento del antagonista (RCA) superiores a los radios de crecimiento del patógeno (RCP). Se observó que el primer contacto entre hifas del antagonista y el patógeno fue de 3 a 4 días en la mayoría de las cepas a excepción de la cepa THU-01 que fue entre 5 a 8 días. Sin embargo, con el papel celofán, solo con la cepa THU-01 se logró observar el traslape de hifas de ambos hongos, ya que para las demás cepas de Trichoderma spp., en la mayoría de los casos, el antagonista no permitió que el patógeno creciera lo suficiente para alcanzar la mitad de la caja Petri, donde se encontraba el papel celofán.

Los mayores radios de crecimiento de las cepas de Trichoderma fueron THC-02 (Colombia), y THM-03 (nativa) con 39,3, y 41,2 mm respectivamente (Figura 3A). Cabe destacar que las cepas del antagonista llegaron a tener un radio de crecimiento muy cercano al de los respectivos tratamientos testigos, de manera que a los 8 días del ensayo, en todos los casos, lograron cubrir totalmente la superficie de la caja Petri con 42,75 $\mathrm{mm}(100 \%)$.
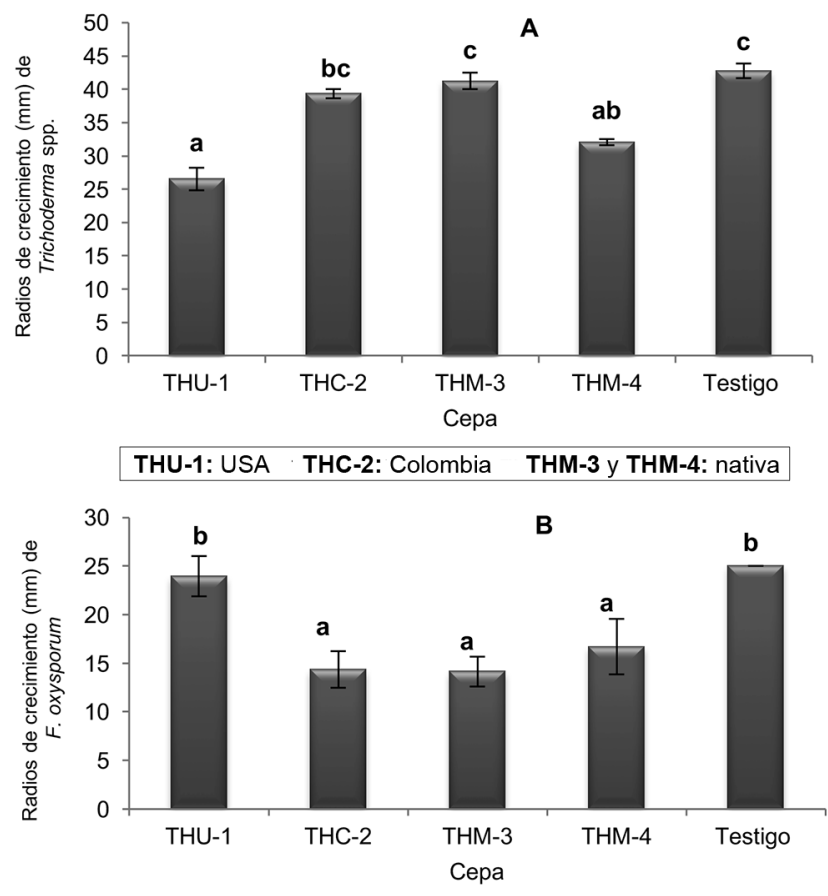

THU-1: USA THC-2: Colombia THM-3 y THM-4: nativa
Figura 3. Radios de crecimiento del antagonista A y del patógeno B, correspondientes a cultivos duales de los diferentes aislamientos de Trichoderma spp.: (THU01 y THM-04: T. guizhouense; THC-02: $T$. asperellum y THM03: $T$. asperelloides) frente a Fusarium oxysporum, al octavo día de evaluación.

Letras diferentes indican diferencias significativas para Tukey $p=0,05$. Las barras verticales representan el error estándar. Todos los testigos alcanzaron el máximo crecimiento. 
Por otra parte, los radios de crecimiento del patógeno (Figura 3B) en casi todos los tratamientos estuvieron por debajo del testigo que alcanzó un crecimiento de 25,05 mm; no obstante, el tratamiento que contenía la cepa THU-01 (proveniente de Estados Unidos) tuvo poco efecto sobre el desarrollo del patógeno con un RCP de 23,95 mm cercano al testigo (Figura 3B) y el menor RCA (Figura 3A).
En la Figura 4 se observan los resultados de la prueba de micoparasitismo, en las cepas THC-02 y THM-04 que lograron alcanzar el grado 4, mientras que THM-03 obtuvo un 3,6, que logró una invasión total de la superficie de la colonia del patógeno y esporular sobre ella, mientras que la THU-01 solo obtuvo 1 grado, que invadió un $1 / 4$ de la superficie de la colonia de F. oxysporum.

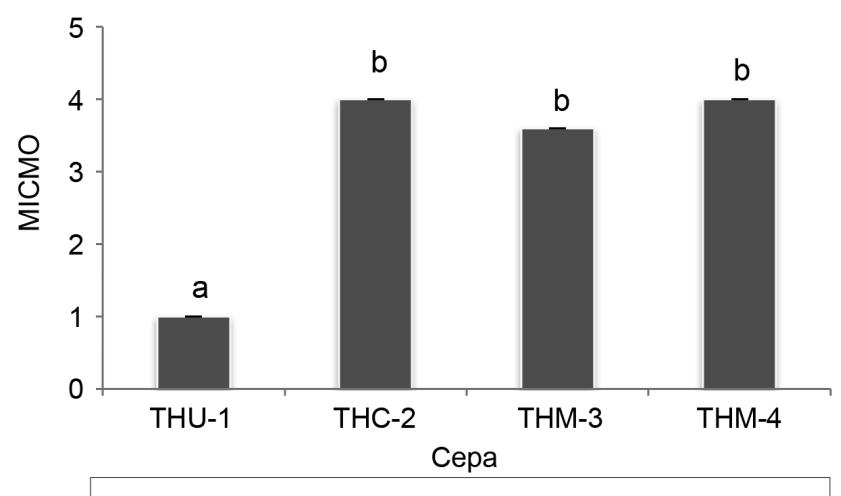

THU-1: USA THC-2: Colombia THM-3 y THM-4: nativa

Figura 4. Grado de micoparasitismo (MICMO) correspondiente a cultivos duales de los distintos aislamientos de Trichoderma spp.: (THU-01 y THM-04: T. guizhouense; THC-02: T. asperellum y THM-03: T. asperelloides.) frente a Fusarium oxysporum, al octavo día de evaluación.

Letras diferentes indican diferencias significativas para Tukey $\mathrm{p}=0,05$.

Las barras verticales representan el error estándar.

Al determinar el porcentaje de inhibición del crecimiento radial (PICR), la mayoría de los tratamientos lograron superar el 50\% de inhibición del crecimiento radial del patógeno $F$. oxysporum (Figura 5). Se encontraron diferencias significativas entre cepas $(\mathrm{p} \leq 0,0001)$, donde las más efectivas fueron THM-03, THC-02, THM-04 con $67 \%, 63 \%, 59 \%$ de inhibición respectivamente, mientras que con el THU-01 solo alcanzó un $44 \%$. 


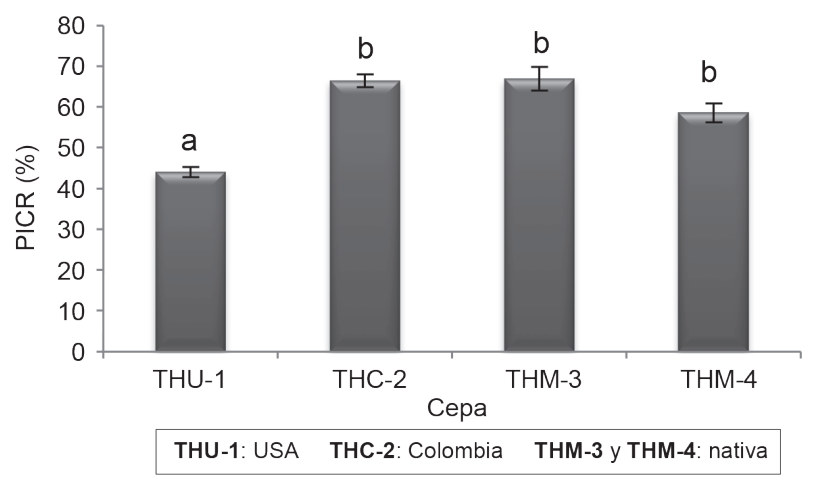

Figura 5. Porcentaje de inhibición del crecimiento radial (PICR), correspondiente a cultivos duales de los diferentes aislamientos de especies de Trichoderma de productos comerciales importados y nativos frente al patógeno Fusarium oxysporum, al octavo día de evaluación.

Letras diferentes indican diferencias significativas para Tukey $\mathrm{p}<0,05$.

Las barras verticales representan el error estándar. THU-01 y THM-04: T. guizhouense; THC-02: T. asperellum y THM-03: $T$. asperelloides.

\section{DISCUSIÓN}

Trichoderma es un grupo monofilético (Kullnig et al. 2002) con teleomorfos en el género Hypocrea o relacionados con Hypocreales. Estudios sobre la conexión entre teleomorfosanamorfos exponen características morfológicas traslapadas entre especies en los anamorfos del género Trichoderma (Chaverri y Samuels 2003). En consecuencia, las diferencias morfológicas no son indicadores fiables del grado de divergencia genética entre las especies y observaciones morfológicas, solas no son suficientes para la identificación precisa de las especies.

En esta investigación se identificaron las diferentes cepas aisladas de productos comerciales importados, así como, 2 cepas aisladas de cultivos en Costa Rica, por lo tanto la cepa THU-1 (Estados Unidos) y la cepa nativa, proveniente de Cartago, THM-04 se identificaron como Trichoderma guizhouense, la cual es una especie del complejo Trichoderma harzianum, que fue descrita por Chaverri et al. (2015). Además, es importante tomar en cuenta que el producto utilizado de Estados Unidos (THU-1) dice contener T. harzianum, sin embargo, según este análisis, la especie no correspondía, lo cual se comparó con el estudio realizado por Chaverri et al. (2015), quienes al revisar la taxonomía de las especies del complejo de Trichoderma harzianum ajustado al factor de elongación 1- $\alpha$ (TEF1) y al analizar cepas de productos comerciales utilizados para biocontrol, determinaron que la mayoría de estos productos presentan una errónea identificación de la especie de Trichoderma, ya que productos como el Promot dice contener T. harzianum cuando en realidad es $T$. guizhouense u otros productos que utilizan la cepa T22 de "T. harzianum" y en realidad es $T$. afroharzianum.

Además, de las características moleculares evaluadas para derterminar especies de Trichoderma, es importante el enfoque ecológico y distribución geográfica de especies, ya que se considera que Trichoderma harzianum se encuentra en Europa y Norte América en hábitats en su mayoría de suelo, también del compost de setas y en ocasiones endótifo en tallos y posiblemente es fungícola. En el caso de $T$. guizhouense es posiblemente cosmopolita y se encuentra en África (Camerún, Ghana), Europa (Croacia, Grecia, Italia y España) y Asia 
(Indonesia, Japón). El hábitat es similar a $T$. harzianum (Chaverri et al. 2015).

En el caso de las cepas THC-02 (Colombia) y la cepa nativa THM-03 (San Carlos) se identificaron morfológicamente como Trichoderma asperellum (Figura 1), sin embargo, al realizar los análisis moleculares de las secuencias del factor de elongación 1- $\alpha$ (TEF1) se determinó la cepa nativa THM-03 como T. asperelloides (Figura 2). En un estudio realizado por Samuels et al. (2010) nombran a esta nueva especie, segregándola a partir de T. asperellum, y aclaran que esta nueva especie no puede distinguirse por su fenotipo, biología o biogeografía y que además, las características morfologícas son muy similares entre las 2 especies, pero es más común encontrar a T. asperellum. Es por ello que todavía se encuentren aislamientos de T. asperellum que no se hayan re-identificado como $T$. asperelloides como se observa en el árbol de las relaciones filogenéticas o que no existe todavía una buena separación de los linajes, ya que posiblemente $T$. asperelloides derivó de T. asperellum. La diversidad sugiere que T. asperellum se originó por recombinación, mientras que $T$. asperelloides parece ser clonal (Mukherjee et al. 2013).

En cuanto a las pruebas de micoparasitismo in vitro, se pudo observar que variaron los tiempos para el contacto entre hifas de las diferentes cepas de Trichoderma spp. y las de $F$. oxysporum; aunque al patógeno se le dio 3 días de ventaja, porque su crecimiento fue más lento. Al comparar con otros estudios, estos resultados son muy similares a los encontrados por Michel (2001), quien encontró valores al primer contacto entre cepas nativas de Trichoderma spp. y $F$. oxysporum (Fo) de 3 a 6 días y con F. subglutinans (Fs) de 3 a 10; mientras que, Solano (2004) de 3 a 4 días con $F$. oxysporum y de 2 a 3 con F. subglutinans. De la misma manera Michel et al. (2005b) mencionan entre 5 y 7 días para el contacto de $T$. harzianum contra $F$. oxysporum f. sp. lycopersici. Según Michel et al. (2009), indican que entre menor sean los días al contacto, es mayor la agresividad que existe por parte del hongo antagonista y menor la resistencia del fitopatógeno. La colonización del área, al competir por espacio y nutrientes, es una manera de ejercer biocontrol, ya que se reduce o se detiene por completo el desarrollo del micelio del otro hongo (Dennis y Webster 1971).

En cuanto a los radios de crecimiento de las colonias, tanto del patógeno como de las cepas del antagonista, se lograron encontrar diferencias significativas $(\mathrm{p} \leq 0,0001)$ entre las cepas de Trichoderma spp. nativas y las importadas de otros países frente al patógeno $F$. oxysporum. El mayor antagonismo corresponde a las cepas THC-02 (T. asperellum) y THM-03 (T. asperelloides) y la cepa de $T$. guizhouense (THM-04) (Figura 3 A y B). Los resultados son similares a los presentados en un estudio de confrontación in vitro de cepas de $T$. harzianum vs. F. solani realizado por Suárez et al. (2008). Este mayor crecimiento por parte de las cepas de Trichoderma podría deberse a que presentaron una mayor tasa de incorporación de nutrientes, metabolismo y un crecimiento superior a $F$. oxysporum, debido a que se utilizaron distintos mecanismos como secreción de enzimas hidrolíticas, entre ellas celulasas, quitinasas, glucanasas, xilasas y muchas veces las proteasas, las cuales pueden estar implicadas en los mecanismos de biocontrol, lo que permite al antagonista aprovechar mejor los nutrientes del medio y privar al patógeno de utilizar los recursos disponibles (Zeilinger y Oman 2007, Vinale et al. 2008, Rojan et al. 2010).

De acuerdo con la clasificación del micoparasitismo, mediante la escala (Figura 4), se demuestra que a nivel de laboratorio tanto Trichoderma asperellum, T. asperelloides como T. guizhouense pueden ejercer su antagonismo sobre Fusarium oxysporum, pero existen diferencias entre cepas de la misma especie $T$. guizhouense, como es el caso de THU-01 (grado 1) y THM-04 (grado 4). Si se compara con otros estudios que emplearon una escala de clasificación de antagonismo similar a la de este estudio de Bell et al. (1982), donde 1=Trichoderma sobrecrece completamente al patógeno y cubre totalmente la superficie del medio, $2=$ Trichoderma 
sobrecrece las 2 terceras partes de la superficie del medio, $3=$ Trichoderma, y el patógeno colonizan cada uno, aproximadamente la mitad de la superficie y ningún organismo parece dominar al otro, 4= el patógeno coloniza las 2 terceras partes de la superficie del medio y parece resistir a la invasión por Trichoderma, y 5= el patógeno sobrecrece completamente a Trichoderma y ocupa la superficie total del medio. En el caso de $F$. solani y F. oxysporum, Michel et al. (2005a) encontraron diferente antagonismo (1, 2 y 3$)$, por lo que se obtuvo menor número de cepas con antagonismo 1 con Fo. En otro ensayo se clasificó el antagonismo de 20 cepas de Trichoderma contra el mismo patógeno y todas resultaron ser clase 1 (Michel 2005b), lo cual corresponde al grado 4 de este ensayo según la escala de Ezziyyani et al. (2004). Al evaluar esas mismas cepas de Trichoderma contra Sclerotium rolfsii Sacc., solo 3 fueron clase 1 y 2 , y en las demás cepas el patógeno fue más agresivo.

Se debe tener presente que el complejo proceso de micoparasitismo se compuso de muchos eventos que surgieron desde el reconocimiento del hospedero, el ataque y la subsecuente penetración y muerte. Durante el proceso, Trichoderma secretó enzimas que degradaron la pared celular del otro hongo. Además, por la secreción de exoenzimas hidrolíticas disminuyó el crecimiento y la actividad del patógeno (Vinale et al. 2008, Brunner et al. 2003, Harman 2006, 2008).

Una vez que los hongos estuvieron en contacto, Trichoderma puede enrollarse alrededor de las hifas del hongo patógeno y formar estructuras especializadas de tipo apresorio, las cuales pueden penetrar en el interior de su adversario. La unión parece estar mediada por la interacción entre carbohidratos de la pared celular de Trichoderma y lectinas del patógeno (Inbar y Chet 1996, Rocha-Ramírez et al. 2002). Una vez en contacto, Trichoderma produce varias CWDEs (Cel Wall Degrading Enzymes) y antibióticos, como los peptaiboles (Schirmböck et al. 1994; Degenkolb et al. 2003), que generarían agujeros en la pared celular. Finalmente, Trichoderma digirió el contenido intracelular del hongo, proceso que fue acompañado de algunos cambios morfológicos como vacuolización, pérdida de citoplasma y desintegración de las hifas del hospedante (Benhamou y Chet 1996). En algunos casos, también se observó la esporulación de Trichodermatras la digestión del otro hongo (Elad et al. 1984).

Al determinar el porcentaje de inhibición del crecimiento radial (PICR), la mayoría de los tratamientos lograron superar el $50 \%$ de inhibición del crecimiento radial del patógeno F. oxysporum (Figura 5). Aunque Trichoderma guizhouense fue menos efectiva para combatir al patógeno, la cepa nativa (THM-04) presentó más eficacia que la importada. Según Ploetz (2004), la especificidad de las especies de Trichoder$m a$, en la eficacia del combate de Fusarium oxysporum, son contradictorios, y la mayoría se refieren a la eficiencia de los ensayos in vitro o invernadero bajo condiciones controladas y por lo tanto, dichos resultados no se pueden generalizar a todas las especies y cepas aisladas de Trichoderma. Además, el antagonismo de las cepas de Trichoderma spp. pueden deberse a que liberan metabolitos secundarios, volátiles y no volátiles, que producen un efecto de antibiosis (Cardoza et al. 2005, Webster y Weber 2007, Shoresh et al. 2010).

En conclusión, se encontraron diferencias morfológicas, pero no genéticas entre las mismas especies nativas de Costa Rica y las importadas en productos comerciales para el hongo Trichoderma spp; por lo tanto, es necesario realizar otros estudios moleculares que permitan comprender las posibles diferencias genéticas intraespecíficas para este género.

En las pruebas in vitro se presentaron diferencias en la capacidad antagónica de las diferentes especies de Trichoderma frente al patógeno Fusarium oxysporum, ya que las cepas de $T$. asperellum, $T$. asperelloides y la cepa nativa de $T$. guizhouense mostraron ser más agresivas en el combate del patógeno, independiente de su procedencia, por lo que se podría ampliar el estudio con más aislamientos. 


\section{LITERATURA CITADA}

Agrios, G. 2005. Plant pathology (en línea). 5ta ed. Elsevier Academic Press. 952 pp. Consultado 15 feb. 2018. DOI: https://doi.org/10.1016/C2009-0-02037-6

Altschul, S; Madden, T; Schäffer, A; Zhang, J; Miller, W; Lipman, D. 1997. Gapped BLAST and PSIBlast: a new generation of protein database search programs. Nucl. Acids. Res. 25:3389-3402.

Arikan, S; Lozano, M; Paetznickv, V; Rex, J. 2001. In vitro susceptibility testing methods for caspofungin against Aspergillus and Fusarium isolates. Antimicrob Agent Chemother 45:327-330.

Bell, D; Well, H; Markham, C. 1982. "In vitro" antagonism of Trichoderma species against six fungal plant pathogens. Phytopathology 72:379-382.

Benhamou, N; Chet, I. 1996. Parasitism of sclerotia of Sclerotium rolfsii by Trichoderma harzianum: ultraestructural and cytochemical aspects of the interaction. Phytopathology 86: 405-416.

Booth, C. 1971. The genus Fusarium. CAB, CMI. England. 237 p.

Brunner, K; Peterbauer, K; Mach, L; Lorito, M; Zeilinger, S; Kubicek,P. 2003. The Nag1 N-acetylglucosaminidase of Trichoderma atroviride is essential for chitinase induction by chitin and of major relevance to biocontrol. Curr Genet 43:289-295.

Burges, B; Ummerell, S; Bullock, K; Gott, J; Backhouse, P. 1994. Laboratory Manual for Fusarium Research. University of Sydney and Botanic Gardens, Department of Crop Science, Fusarium Research Laboratory, Sydney, Australia. 191 p.

Caravaca, P. 2008. Boletín 1: Tomate de Costa Rica (en línea). San José, Costa Rica. Subgerencia de Desarrollo Agropecuario, Dirección General Mercadeo y Agroindustria. Consultado 15 feb. 2018. Disponible en http://www.mercanet.cnp.go.cr

Cardoza, E; Hermosa, R; Vizcaíno, A; Sanz, L; Monte, E; Gutiérrez, S. 2005. Secondary metabolites produced by Trichoderma and their importance in the biocontrol process. In Mellado, E; Barredo, J (eds.). Microorganisms for Industrial Enzymes and Biocontrol. Kerala, India, Research Signpost. p. 1-22

Chairman, E; Bird, G; Fisher, K; Hickey, K; Lewis, F; Line, R; Rickard, S. 1978. Methods for evaluating plant fungicides, nematicides and bactericides. The American Phytopathological Society. Minneapolis, USA. $141 \mathrm{p}$.

Chaverri, P; Samuels, G. 2003. Hypocrea/Trichoderma (Ascomycota, Hypocreales, Hypocreaceae): species with green ascospores. Stud. Mucol. 48:1-116.

Chaverri, P; Branco, F; Jaklitsch, W; Gazis, R; Degenkolb, T; Samuels, G. 2015. Systematic of the Trichoderma harzianum species complex and the re-identification of commercial biocontrol strains. Mycología 107(3):558-590.

Chérif, M; Benhamou, N. 1990. Cytochemical aspects of chitin breakdown during the parasitic action of a Trichoderma sp. on Fusarium oxysporum f. sp. radicis-lycopersici. Phytopathology 80:1406-1414.

Chet, I; Benhamou, N; Haran, S. 2002. Mycoparasitism and lytic enzymes. In Kubicek, CP; Harman, GE (eds.). Trichoderma and Gliocladium (vol. 2). Londres, Reino Unido, Taylor y Francis. p.153-172.

Córdoba, M. 2003. Biocontrol de Fusarium oxysporum f. sp. lycopersici por Trichozam ${ }^{\circledR}$ (Trichoderma harzianum) y Mycoral ${ }^{\circledR}$ (micorriza vesículo arbuscular) en el cultivo de tomate. Tesis Lic. Tegucigalpa, Honduras, Universidad Zamorano. 43 p.

De la Cruz, E; Ruepert, C; Wesseling, C; Monge, P; Chaverri, F; Castillo, L; Bravo, V. 2004. Los plaguicidas de uso agropecuario en Costa Rica: impacto en la salud y el ambiente. Informe de consultoría para Área de Servicio Agropecuario y Medio Ambiente de la Contraloría General de la República. Heredia: IRET, Universidad Nacional Autónoma. 95 p.

De la Cruz, J; Rey, M; Lora, JM; Hidalgo-Gallego, A; Domínguez, F; Pintor-Toro, JA; Llobell, A; Benítez, T. 1993. Carbon source control on ß-glucanase, chitobiase and chitinase from Trichoderma harzianum. Arch. Microbiol. 159:316-322.

Degenkolb, T; Berg, A; Gams, W; Schlegel, B; Grafe, U. 2003. The occurrence of peptaibols and structurally related peptaibiotics in fungi and their mass spectrometric identification via diagnostic fragmentions. J. Pept. Sci. 9:666-678.

Dennis, C; Webster, J. 1971. Antagonistic properties of species-groups of Trichoderma. III: Hyphal interactions. Trans. Brit. Mycol. Soc. 57:363-369.

Elad, Y; Barak, R; Chet, I. 1984. Parasitism of esclerotia of Sclerotinium rolfsii by Trichoderma harzianum. Soil. Biol. Biochem. 16:381-386.

Elad, Y; Chet, I; Hennis, Y. 1982. Degradation of plant pathogenic fungi by Trichoderma harzianum. Can. J. Microbiol. 28:719-725.

Ellis, D. 2006. The University of Adelaide Australia (en línea). Estados Unidos. Consultado 22 ago. 2018. Disponible en http://www.mycology.adelaide.edu. au/Fungal_Descriptions

Ezziyyani, M; Pérez, S; Requena, M; Rubio, L; Candela, M. 2004. Biocontrol por Streptomyces rochei-Ziyani-, de la podredumbre del pimiento (Capsicum annuum L.) causada por Phytophthora capsici. Ann. de Biol. 26:69-78.

Geiser, D; Jiménez, M; Kang, S; Makalowska, I; Veeraraghavan, N; Ward, T; Zhang, N; Kuldau, G; O'Donnell, K. 2004. FUSARIUM-ID v. 1.0: A DNA sequence database for identifying Fusarium. Europ. J. Plant. Pathol. 110:473-479. 
Gilchrist, L; Fuentes, C; Martínez, R; López, E; Duveiller, R; Singh, M; Henry, I; García, A. 2005. Guía práctica para la identificación de algunas enfermedades de trigo y cebada. 2 ed. México, D. F. CIMMYT. 260 p.

Gupta, V; Schmoll, M; Herrera, A; Upadhyay, R; Druzhinina, I; Tuohy, M. 2014. Biothecnology and biology of Trichoderma. New York, Estados Unidos, Elsevier. 549 p.

Hall, T. 1999. BioEdit: a user-friendly biological sequence alignment editor and analysis program for Windows 95/98/NT. Nucleic Acids Symp. Series 41:95-98.

Harman, GE. 2006. Overview of mechanism and uses of Trichoderma spp. Phytopathology 96:190-194.

Harman, GE; Bjorkman, T; Ondik, K; Shoresh, M. 2008. Changing paradigms on the mode of action and uses of Trichoderma spp. for biocontrol. Outl. Pest. Manag. 19:24-29.

Hibar, K; Daami-Remadi, M; Khiareddine, H; El-Mahjoub, M. 2005. Effet inhibiteur in vitro et in vivo du Trichoderma harzianum sur Fusarium oxysporum f. sp. radicis-lycopersici. Biotech. Agron. Soc. Environ. 9:163-171.

Hibar, K; Daami-Remadi, M; Hamada, W; El-Mahjoub, M. 2006. Bio-fungicides as an alternative for tomato Fusarium crown and root rot control. Tunisian J. Plant. Protec. 1:19-29.

Howell, C. 2003. Mechanisms employed by Trichoderma species in the biological control of plant diseases: the history and evolution of current concepts. Plant. Dis. 87:4-10.

Inbar, J; Chet, I. 1996. The role of lectins in recognition and adhesion of the mycoparasitic fungus Trichoderma spp. to its host. Adv. Exp. Med. Biol. 408: 229-231.

Jiménez, C; Sanabria, A. 1997a. Evaluaciones in vitro de siete fungicidas para el control de Fusarium oxysporum f. sp. lycopersici. Fitop. Venez. 10(1):32-37.

Jiménez, C; Sanabria, A. 1997b. Control biológico in vitro de Fusarium oxysporum f. sp. lycopersici. Fitop. Venez. 10(1):38-42.

Komoda, H. 1975. Development of a selective medium for quantitative isolation of Fusarium oxysporum from natural soil. Rev. Plant. Res. 8:114-125.

Kullnig, C; Szakacs, G; Kubicek, C. 2002. Phylogeny and evolution of the fungal genus Trichoderma - a multigene approach. Mycol. Res. 106:757-767.

Lu, Z; Tombolini, R; Woo, S; Zeilinger, S; Lorito, M; Jansson, JK. 2004. In vivo study of Trichodermapathogen-plant interactions, using constitutive and inducible green fluorescent protein reporter systems. App. Environ. Microbiol. 70:3073-3081.

Michel, A; Rebolledo, O; Lezama, R; Ochoa, M; Mesina, J; Samuels, G. 2001. Especies de Trichoderma en suelos cultivados con mango afectados por "escoba de bruja" y su potencial inhibitorio sobre Fusarium oxysporum y $F$. subglutinans. Rev. Mex. Fitop. 19:154-160.

Michel, A; Otero, M; Rebolledo, O; Lezama, R; Ariza, R; Barrios, A. 2005a. Producción y efecto antagónico de quitinasas y glucanasas por Trichoderma spp., en la inhibición de Fusarium subglutinans y Fusarium oxysporum in vitro. Rev. Chapingo, Serie Horticultura 11:273-278.

Michel, A; Reyes, A; Otero, M; Rebolledo, O; Lezama, R. 2005b. Potencial Antagónico de Trichoderma spp. sobre Fusarium oxysporum Schlechtend.:Fr. f. sp. lycopersici (Sacc.) Snyder y Hansen y Sclerotium rolfsii (Sacc.) in vitro e invernadero. Rev. Mex. Fitop. 23:284-291.

Michel, A; Otero, M; Solano, L. 2009. Biocontrol in vitro con Trichoderma spp. de Fusarium subglutinans (Wollenweb y Reinking) Nelson, Toussoun y Marasas y F. oxysporum Schlecht., agentes causales de la "Escoba de Bruja" del Mango (Mangifera indica L.). Rev. Mex. Fitop. 27(1):18-26.

Montealegre, J; Herrera, R; Velásquez, J; Silva, P; Besoaín, X; Pérez, L. 2005. Biocontrol of root and crown rot in tomatoes under greenhouse conditions using Trichoderma harzianum and Paenibacillus lentimorbus. Additional effect of solarization. J. Biotech. 8(3):249-257.

Nelson, EB. 1991. Current limits to biological control of fungal phytopathogens. In Arora, DK; Rai, B; Mukerji, KG; Knudsen, GR (eds.). Handbook of Applied Mycology. New York, USA. p. 327- 355.

O’Donnell, K; Kistler, H; Cigelnik, E; Ploetz, R. 1998. Multiple evolutionary origins of the fungus causing Panama disease of banana: Concordant evidence from nuclear and mitochondrial gene genealogies. Proc. of the Nat. Acad. of Scien. of the USA. 95:2044-2049.

Olmedo, V; Casas, S. 2014. Molecular mechanisms of biocontrol in Trichoderma spp. and their applications in agriculture. In Gupta et al. (eds.). Biothecnology and biology of Trichoderma. Elsevier, Estados Unidos. p. 429-454.

Papavizas, GC. 1985. Trichoderma and Gliocladium: biology, ecology and potential for biological control. Annu. Rev. Phytopathol. 19:332-349.

Ploetz, RC. 2004. Fusarium wilt of banana is caused by several pathogens referred to as Fusarium oxysporum f. sp. cubense. Phytopathology 96:653-656.

Rocha-Ramírez, V; Omero, C; Chet, I; Horwitz, BA; Herrera-Estrella, A. 2002. Trichoderma atroviride G-protein alpha-subunit gene tgal is involved in mycoparasitic coiling and conidiation. Eukaryot. Cell. 1:594-605.

Rojan, J; Tyagi, R; Prévost, D; Brar, S; Pouleur, S; Surampalli, R. 2010. Mycoparasitic Trichoderma viride as a biocontrol agent against Fusarium oxysporum f. sp. 
adzuki and Pythium arrhenomanes and as a growth promoter of soybean. Crop. Prot. 29:1452-1459.

Samuels, G; Chaverri, P; Farr, D; McCray, E. 2013. Trichoderma online, systematic mycology and microbiology Laboratory, ARS, USDA (en línea). Estados Unidos. Consultado 28 jul. 2018. Disponible en /taxadescriptions/keys/TrichodermaIndex.cfm

Samuels, G; Ismaiel, A; Bon, M; Respinis, S; Petrini, O. 2010. Trichoderma asperellum sensu lato consists of two cryptic species. Mycología 102(4):944-966.

Sanz, L; Montero, M; Grondona, I; Vizcaíno, JA; Llobell, A; Hermosa, R; Monte, E. 2004. Cell-wall degrading isoenzyme profiles of Trichoderma biocontrol strains show correlation with rDNA taxonomic species. Curr. Genet. 46:277-286.

Sanz, L; Montero, M; Redondo, J; Llobell, A; Monte, E. 2005. Expression of an alpha-1,3- glucanase during mycoparasitic interaction of Trichoderma asperellum. FEBS. J. 27(2):493-499.

Schirmböck, M; Lorito, M; Wang, YL; Hayes, CK; ArisanAtac, I; Scala, F; Harman, GE; Kubicek, CP. 1994. Parallel formation and synergism of hydrolytic enzymes and peptaibol antibiotics, molecular mechanisms involved in the antagonistic action of Trichoderma harzianum against phytopathogenic fungi. App. Environ. Microbiol. 60:4364-4370.

Seifert, K. 1996. Fuskey. Fusarium interactive key (en línea). Agriculture and agifood Canada. Consultado: 20 ago. 2017. Disponible en http://www.ctu.edu.vn/ colleges/agri/gtrinh/fuskey.pdf

Shoresh, M; Harman, G; Mastouri, F. 2010. Induce systematic resistance and plant responses to fungal biocontrol agents. Annu. Rev. Phytopathol. 48:21-43.

Shoukouhi, P; Bissett, J. 2008. PReferred primers for sequencing the $5^{\prime}$ end of the translation elongation factor 1-alpha gene (eEF1a1) (en línea).
Consultado 28 jul. 2018. Disponible en http:// www.isth.info/methods

Solano, P. 2004. Control biológico de la escoba de bruja del mango con Trichoderma spp. Tesis Lic. Guerrero, México. Colegio Superior Agropecuario del Estado de Guerrero, Centro de Estudios Profesionales. 82 p.

Suárez, C; Fernández, R; Valero, N; Gámez, R; Páez, A. 2008. Antagonismo in vitro de Trichoderma harzianum Rifai sobre Fusarium solani (Mart.) Sacc., asociado a la marchitez en maracuyá. Rev. Colom. Biotec. 10(2):35-43.

Tamura, K; Stecher, G; Peterson, D; Filipski, A; Kumar, S. 2013. MEGA 6: molecular evolutionary genetics analysis version 6.0. Molec. Biol. Evol. 30:2725-2729.

Thompson, J; Higgins, D; Gibson, T. 1994. CLUSTAL W: improving the sensitivity of progressive multiple sequence alignment through sequence weighting, position-specific gap penalties and weight matrix choice. Nucl. Acids. Res. 22:4673-4680.

Vinale, F; Sivasithamparam, K; Ghisalberti, E; Marra, R; Woo, S; Lorito, M. 2008. Trichoderma-plantpathogen interactions. Soil Biol. Biochem. 40:1-10.

Viterbo, A; Montero, M; Ramot, O; Friesem, D; Monte, E; Llobell, A; Chet, I. 2002a. Expression regulation of the endochitinase chit36 from Trichoderma asperellum (T. harzianum T-203). Curr. Genet. 42:114-122.

Viterbo, A., Ramot, O., Chemin, L., Chet, I. 2002b. Significance of lytic enzymes from Trichoderma spp. in the biocontrol of fungal plant pathogens. Antonie Van Leeuw. 81:549-556.

Webster, J; Weber, R. 2007. Introduction to Fungi. $3^{\text {er }}$ ed. Cambridge, UK, Cambridge University Press. $841 \mathrm{p}$.

Zeilinger, S; Oman, M. 2007. Trichoderma biocontrol: signal transduction pathways involved in host sensing and mycoparasitism. Gene Regul. Syst. Biol. 1:227-234. 
